REY \& SAINZ-RABANAL / Arte comunitario como herramienta de inclusión: experiencias en el Taller de Expresión Artística del Centro Penitenciario Madrid IV de Navalcarnero

\title{
Arte comunitario como herramienta de inclusión: experiencias en el Taller de Expresión Artística del Centro Penitenciario Madrid IV de Navalcarnero
}

\author{
Community art as an inclusion tool: experiences in the Art \\ Expression Workshop Prison Centre"Madrid IV" of Navalcarnero
}

\author{
Nuria REY. Pontificia Universidad Católica de Ecuador Sede Esmeraldas (Ecuador). \\ nur.rey.nrs@gmail.com \\ Laura DELGADO-GUILLÉN. Universidad Complutense de Madrid (España). \\ lauradelguillen@gmail.com \\ Jorge FERNÁNDEZ-CEDENA. Universidad Complutense de Madrid (España). \\ jorge.f.cedena@gmail.com \\ Silvia SAINZ-RABANAL. Universidad Complutense de Madrid (España). \\ silvia.sainz.rabanal@gmail.com
}

Este artículo recoge descripciones del estudio sobre acciones artístico-educativas en centros penitenciarios en España, caso del Taller de Expresión Artística en el Centro Penitenciario Madrid IV financiada por Solidarios para el Desarrollo.

Resumen: La Educación Artística, como herramienta para la integración social, puede funcionar como canal mediador en diferentes contextos para fomentar el pensamiento crítico y localizar lugares de encuentro e intercambio de perspectivas. En España se han desarrollado varios proyectos en los que esta disciplina se ha incluido en los programas educativos de prisiones para contribuir a la reinserción y el enriquecimiento cultural de los internos. En el presente artículo se expone la experiencia del taller de arte en el Centro Penitenciario Madrid IV, prisión ubicada en Navalcarnero, dentro de la Comunidad de Madrid. Esta iniciativa es coordinada por la asociación Solidarios para el Desarrollo, que trabaja dentro de la acción social y comunitaria con el objetivo de crear espacios que propicien el acercamiento socio-cultural. Se abordan en primer lugar los antecedentes existentes, para después profundizar en la actividad desde la primera toma de contacto en la intervención 
didáctica hasta la materialización y valoración de la actividad, dividendo la experiencia en dos bloques que explican tanto la acción desde la parte social como desde la parte artística. Los resultados que derivarán en conclusiones se obtienen a partir de las fichas de seguimiento de objetivos, así como de la extracción de categorías recogidas en la sesión de evaluación. Así, esta experiencia puede convertirse en un referente para las prácticas artísticas en contextos de privación de libertad como herramienta de mediación y reflexión comunitaria.

Palabras clave: arte comunitario, centro penitenciario, educación artística, mediación, inclusión social, taller.

\begin{abstract}
Art Education, as a tool for social integration, is able to work as a mediator in different contexts to enhance critical thinking, find out meeting points, and exchange perspectives. Spain has developed several projects in which this discipline has been included in education programs in prison centres in order to contribute to the reintegration and cultural enrichment of inmates. This text describes the experience in the art workshop at the Prison Centre Madrid IV, located at Navalcarnero, Madrid.This iniciative has been coordinated by the organization'Solidarios para el Desarrollo', which works within the social and community action with the aim of creating spaces that promote rapprochement between different cultures and social situations. This article analyses the existing background, and it then deepens in the experience from the first contact until the accomplishment and evaluation of the activity. It is divided in two main parts that explain the social action and the artistic intervention. The results, which will end up in conclusions, have been obtained through the tracking cards of the targets, and the categories gathered in the assessment session. In this way, this experience can become a referent in the task of bringing artistic practices to deprivation of liberty contexts, using it as a tool for mediation and community reflection.
\end{abstract}

Keywords: community art, prison, art education, mediation, social inclusion, workshop

\title{
Introducción
}

El arte, desde su carácter mediador, se ha demostrado una herramienta que invita a la reflexión, la experiencia y la producción común, pudiendo ofrecer espacios transformadores de libertad y cuestionamiento. El texto que se presenta recoge la experiencia del Taller de Expresión Artística desarrollado en el año 2015 en el Centro Penitenciario Madrid IV, localizado en el municipio madrileño de Navalcarnero.

La puesta en marcha de este taller comienza gracias al apoyo de la asociación Solidarios para el Desarrollo, que interviene en contextos de exclusión mediante el voluntariado y la acción social. Su objetivo principal es el de trabajar activamente con personas de colectivos vulnerables a través de la comunicación y la acción 
social, produciendo diferentes niveles de impacto en la transformación de realidades sociales.

El planteamiento inicial de este taller consiste en conectar mediante las estrategias artísticas el mundo de fuera y de dentro del centro, buscando un acercamiento entre experiencias y sensaciones, siempre dentro de los numerosos límites de trabajar en una prisión hermética por cuestiones de seguridad y control. Ejemplo de ello son el estricto horario para realizar los talleres, así como el tiempo, que en ocasiones se ve reducido por la espera en controles de acceso, o los materiales, que a veces son retirados a la entrada del taller por considerarse inadecuados. Por todo ello, se debe abordar la programación del taller desde la flexibilidad.

Inmersos en la acción social y comunitaria, se considera fundamental determinar un objetivo general que se centre en el desarrollo de la actividad artística, que finalmente se convierte en la excusa para propiciar entornos de libertad grupal y construcción colectiva de aprendizajes. Este objetivo fue el de ampliar el imaginario hacia propuestas y producciones de arte contemporáneo y análisis de la cultura visual, rompiendo con las arraigadas expectativas de que un taller de arte es igual a un taller de artesanías y decoraciones.

Tras la revisión de literatura y antecedentes, se explicará el desarrollo del taller, los resultados y las conclusiones extraídas.

\section{Arte comunitario para la inclusión social}

\section{- Acción social y comunitaria: las relaciones humanas del taller}

El trabajo comunitario, más en contextos que podrían considerarse excluidos como es un centro penitenciario, conlleva un trabajo social y humano que provoca situaciones intensas entre los diferentes miembros. La labor en el Taller de Expresión Artística quedaría enmarcada dentro de las prácticas que podríamos relacionar con los modos de hacer del arte comunitario:

el término arte comunitario se asocia a un tipo de prácticas que buscan una implicación con el contexto social, que persiguen, por encima de unos logros estéticos, un beneficio o mejora social y sobre todo, que favorecen la colaboración y la participación de las comunidades implicadas en la realización de la obra. (Palacios, 2009, p. 199).

La labor como educadores consiste principalmente en organizar y orientar el trabajo de los participantes. Las limitaciones del entorno y el carácter educativo de la actividad remarcan una de las características esenciales de cualquier práctica artística colaborativa: más allá del valor estético de los productos, se hace especial hincapié en el proceso y la colaboración necesaria para la obtención de resultados. 
A través de tales expresiones creativas, los individuos son dotados, adquiriendo paulatinamente voz, visibilidad y la conciencia de formar parte de una totalidad mucho mayor. Lo personal, de este modo, pasa a ser político y el cambio se hace posible, incluso si inicialmente sólo se produce en el interior de la comunidad o en la conciencia pública (Felshin, 2001, p. 76).

Recuperando los estudios de Moreno (2012), se determina que los talleres artísticos en contexto carcelario permiten:

- Romper con la deshumanización instalada en la subcultura de los presos.

- Reforzar la autoestima y el enriquecimiento espiritual.

- Facilitar la toma de conciencia y el proceso de reinserción social.

- Dignificar la vida de las personas.

- Desarrollar cohesión grupal y vínculos afectivos.

- Proyectarse en el futuro de forma más autónoma e integrada.

Las investigaciones del Observatorio del Tercer Sector de Bizkaia (2012) insisten en que el arte se convertiría en el instrumento para la participación y la transformación social en situaciones de exclusión, siendo las personas vulnerables las protagonistas de los procesos de creación.

Una dinámica que aparece en estos contextos, y que también se dio en este taller en concreto, es la jerarquía y asimilación de roles dentro del grupo de participantes. En relación a la resolución de conflictos por cuestiones de liderazgo, García define esta situación de la siguiente manera:

(...) un ambiente donde la "prisionalización" es la norma no escrita, y donde una amplia mayoría se rige por códigos de silencios y predomina la ley del más fuerte. Por ello, trata de experiencias y actividades que son parte vital, de esperanza y de libertad a través del arte en sus diversas formas, y de transformación, que fluctúan entre las personas que viven en régimen penitenciario, y les ayuda a evitar un elevado riesgo de exclusión social y cultural, favoreciendo un punto de encuentro en la sociedad (García, 2014, p. 13)

\section{- Actividad cultural en prisiones: construcción colectiva de otra forma de mirar}

De forma oficial a nivel estatal, encontramos la definición de la persona interna en el Artículo 25.2 de la Constitución Española, según la cual "las penas privativas de libertad y las medidas de seguridad estarán orientadas hacia la reeducación y reinserción social". De la misma manera, el Reglamento Penitenciario (Real Decreto 190/1996) se ocupa en su capítulo tercero de la formación cultural y social de los internos. Concretamente en el artículo 131 de la sección quinta, se indica en varios puntos que será el Centro Directivo del organismo el que programará las actividades referentes al ámbito cultural, pudiendo a su vez los propios internos proponer otras actividades. La realidad es que en la mayoría de los casos estas actividades dependen casi en exclusiva de organizaciones sociales externas al centro. 
La educación artística se considera más bien asociada a unos beneficios personales del interno que no todos los centros creen necesario tratar y que ni siquiera el preso los encuentra interesantes para su futuro personal o laboral. De esta manera, el arte se encuentra en la obligación de permanecer como actividad recreativa o de ocio, junto a otras actividades de esparcimiento dentro del tiempo libre del interno (Villamarín, 2004, p. 16)

La mayoría de los proyectos en relación al arte y la cultura que se desarrollan en las cárceles tienen que ver con una visión del arte de manera tradicional: se promueve la realización de objetos que tengan una funcionalidad, siempre con el objetivo de "ocupar el tiempo" del interno. Según recoge El sistema penitenciario español (2010, p.39), los talleres culturales más habituales en prisiones son "de música, de teatro, de hilos, de pintura, de marquetería y de dibujo, aunque se tienen presentes otros más innovadores, como el de reciclaje, audiovisuales, estampación, esmaltes, radio, etc". En ese sentido, la propuesta de este taller trata de romper con esas ideas, siguiendo las líneas de Ruiz y Vidal:

Las actividades tienen que estar basadas en la calidad artística y de contenidos, al igual que lo estaría cualquier otra actividad cultural o artística en el exterior de la cárcel. No podemos concebir nuestra actividad como un pasatiempo o una actividad meramente lúdica, tenemos que afrontarla con rigor y plantearla como una oportunidad formativa y como un proceso de aprendizaje, lo cual no significa que no puedan tener además un componente lúdico y/o de ocio (2014, p.58).

Las finalidades de estas iniciativas, según (Aguilar, González y Romero, 2009, p.3) se derivan en dos vertientes: "uno hacia el interior, sanear el ambiente, ayudar a curarse al interno, motivarle a comenzar una vida nueva; y otro hacia el exterior, el crear lazos de comunicación con las instituciones sociales".

En España la figura del educador artístico en contextos penitenciarios únicamente está reconocida de forma oficial en Cataluña (Cuesta, 2014). En el caso de este taller, los educadores adoptan el rol de intermediarios, intentando, a través del arte, alcanzar una meta más allá de la creación artística, como señala Hidalgo (2011, p.136) cuando indica que estas iniciativas deben "articular un proyecto más amplio que ponga en contacto a educadores, artistas, sociólogos terapeutas y otros trabajadores socio-culturales con una política cultural integral".

\section{- Proyectos antecedentes de trabajos artísticos en prisiones españolas}

Se pueden mencionar como antecedentes la acción de la Comisión Ciudadana AntiSida de Álava, con más de una década de experiencia en su proyecto para el Centro Penitenciario de Álava, en el que un grupo de voluntarios dirige un taller de pintura con mujeres internas (Burgo, 2015), así como el taller artístico que La Rueda Invertida realizó en el Centro Penitenciario Soto del Real en 2014, culminando con la exposición (PAUSA). Diálogos desde el Módulo 10 en el Museo de Artes Decorativas de Madrid 
De la misma manera, existen varios proyectos en España incentivados por instituciones culturales y museos. Desde 2007 hasta 2011 se realizó un programa de talleres y ponencias por parte del MUSAC de León bajo el nombre de Proyecto Hipatia con un colectivo de mujeres internas, que incluyó la publicación de una revista en la que éstas participaban (Sola, 2014).

En 2008 existe el ejemplo de un grupo de internos del Centro Penitenciario de Mallorca, que colaboró en los proyectos dentro del programa Relat@s, promovido desde la Fundació Pilar i Joan Miró (Revuelta y Arrizabalaga, 2014). En el mismo año, y gracias a la misma fundación, se creó un proyecto por parte de las artistas Patricia Gómez y María Jesús González con los internos de la antigua Prisión de la Palma, funcionando a modo de residencia artística dentro del centro y trabajando estrategias del arte contemporáneo (Gómez \& González, 2014).

En 2010 se realizaron dos proyectos interesantes como referentes. En primer lugar, el que realizó Artium en el Centro Penitenciario de Nanclares de Oca bajo el nombre Aprender a mirar: una introducción al arte contemporáneo, con el que se pretendía acercar el arte contemporáneo y la propia institución museística a los internos (Machín y Talayero, 2014). Por otro lado, se desarrolló el proyecto Mi Caja, Tu refugio en el Centro Penitenciario de Pamplona I gracias al Museo Oteiza, el cual pretendía acercar la figura del artista a los reclusos (Urtasun, 2014).

Entre 2011 y 2012 se creó un proyecto gracias al Centro LABoral (Asturias) llamado TV-LAB ADSIS. Los Kinze en el C. P. de Villabona, con el fin de fomentar la colaboración y el aprendizaje creativo a través de medios de comunicación como la televisión o internet (Arias, Miracle y Pérez, 2014). Por último, se puede mencionar el proyecto ENREDARTE de la Red Provincial Museística de Lugo en el año 2013, que incluye una iniciativa con el Centro Penitenciario de Bonxe (Lugo), y que tuvo como objetivo acercar el museo a personas que no son usuarios de este espacio (Lago, 2014).

Caso aparte sería el del Colectivo Moraga, formado por trabajadores y reclusos del C.P. de Alhaurín de la Torre, en Málaga. El colectivo se formó en 1996 gracias al empeño de los educadores del centro, que convencidos de las virtudes educativas y terapéuticas del arte decidieron poner en funcionamiento un taller de arte permanente en la prisión. Uno de sus objetivos primordiales es el de cambiar conceptos y estereotipos relacionados con la vida tras los muros, con la colaboración de distintas instituciones y galerías en las que exponen regularmente sus trabajos.

\section{Objetivos y métodos del taller}

Para determinar el primer objetivo del taller, el grupo de educadores se apoya en las referencias de Rodrigo (2007, p.77) cuando señala que la educación artística 
debe tener como meta "la justicia social, la promoción de una ciudadanía activa y la construcción de sociedades más democráticas", así como en los argumentos de Ruiz y Vidal (2014) al señalar que es necesario en estas iniciativas trabajar por transmitir una imagen positiva hacia el exterior. Con estas aportaciones, y partiendo de la visión de Solidarios para el Desarrollo, se establece un primer objetivo general, acorde al planteamiento del proyecto artístico-educativo:

- Realizar actividades que conecten el mundo de fuera y de dentro del centro, a partir de las estrategias artísticas.

Gracias al primer contacto con los internos, en el que los educadores conocen los intereses de los mismos, se determinó un segundo objetivo general para las actividades programadas:

- Ampliar el imaginario hacia propuestas y producciones de arte contemporáneo y análisis de la cultura visual.

El primer objetivo atiende a las demandas sociales de la acción educativa, mientras que el segundo se concentra en el sentido artístico y creativo del proyecto.

El taller, que se enmarca dentro de las iniciativas de arte comunitario o educación artística para la inclusión social, es planteado a nivel ético desde las consideradas "buenas prácticas". Para ello existen una serie de indicadores, recogidos por López (2015), que se relacionan con este taller de la siguiente manera:

Tabla 1

Indicadores de buenas prácticas en proyectos de arte comunitario.

\begin{tabular}{ll}
\hline Salud y bienestar & $\begin{array}{l}\text { Apoyo en las sesiones del taller al grupo de } \\
\text { participantes en situación de vulnerabilidad, en } \\
\text { cuestiones de autoestima y estima de las capacidades } \\
\text { individuales }\end{array}$ \\
\hline Cohesión social & Fomento del trabajo en equipo y del compañerismo \\
\hline Empoderamiento de la comunidad & $\begin{array}{l}\text { Fomento de las capacidades creativas y organizativas } \\
\text { del grupo de participantes para la producción creativa }\end{array}$ \\
\hline Identidad & $\begin{array}{l}\text { Desarrollo de sentimiento de pertenencia en el } \\
\text { espacio/tiempo del taller. Cuestionamiento del } \\
\text { futuro. Mejora de la percepción de estos programas } \\
\text { culturales y compromiso por parte de la institución } \\
\text { penitenciaria }\end{array}$ \\
\hline Cambio de perspectiva & $\begin{array}{l}\text { Aumento de la sensación de capacidad creativa por } \\
\text { parte de los internos y amplitud del imaginario de } \\
\text { producción artística }\end{array}$ \\
\hline
\end{tabular}




\begin{tabular}{ll}
\hline Mejora de vida & $\begin{array}{l}\text { Reducción del dolor o la frustración y aumento de } \\
\text { la felicidad individual. Fomento de la alfabetización } \\
\text { visual }\end{array}$ \\
\hline Implicación local & $\begin{array}{l}\text { Contribución del taller al intercambio entre } \\
\text { participantes, no participantes y familiares sobre los } \\
\text { contenidos y producciones realizadas. Intervención } \\
\text { del espacio/sala del taller }\end{array}$ \\
\hline Desarrollo personal & $\begin{array}{l}\text { Búsqueda y experimentación de nuevas habilidades } \\
\text { y desarrollo de habilidades creativas y comunicativas }\end{array}$ \\
\hline Creación de arte público & $\begin{array}{l}\text { Aumento de objetos artísticos en el interior del centro } \\
\text { y conexiones de esos productos dentro-fuera de la } \\
\text { institución }\end{array}$ \\
\hline
\end{tabular}

Nota: indicadores recuperados por López (2015), a partir de los ítems establecidos por la Health Development Agency en el año 2000.

La metodología con la que se plantea el taller es, en un primer momento, flexible y abierta a la escucha y el intercambio de perspectivas con los participantes. Gracias a las primeras sesiones,se puede establecer un esquema de intervención de cada sesión, dividido en las siguientes fases:

- Fase teórica, de presentación de contenidos

- Fase práctica, de producción creativa individual o grupal

- Fase de valoración, para intercambiar opiniones y aprendizajes de cada actividad Uno de los instrumentos de evaluación del taller fue un cuaderno de bitácora, con el que uno de los educadores recogía las valoraciones y observaciones significativas de cada sesión, de cara a la mejora de las sesiones futuras. En ese cuaderno se recogen, a modo de categorías, las valoraciones de los internos con respecto al taller. Otro instrumento utilizado fue la ficha de seguimiento de actividades, con el fin de comprobar el cumplimiento de objetivos del proyecto.

\section{Desarrollo general del taller}

El taller se lleva a cabo por un grupo de seis educadores, en ese momento estudiantes del Máster en Educación Artística en Instituciones Sociales y Culturales de la Universidad Complutense de Madrid (UCM), que comienzan la actividad como voluntarios de la asociación antes mencionada. Este fue un hecho relevante, ya que varios comentarios por parte de los participantes iban encaminados al agradecimiento de desarrollar actividades de esta manera, y no con educadores "convencionales", que tienen una labor más relacionada con el análisis psicológico y de conducta. El desarrollo de esta iniciativa fue posible gracias al convenio entre Solidarios para el Desarrollo y el Departamento de Didáctica de las Artes Plásticas de la Facultad de Bellas Artes (UCM). El taller tuvo una duración de 20 sesiones, un día a la semana, de febrero a junio de 2015, dentro del Módulo de Respeto del C.P. Madrid IV de 
Navalcarnero, lo que supone trabajar con internos que van a terminar su condena, con opciones a continuar sus estudios, participar en las distintas actividades y tener un trabajo dentro del centro.

La primera jornada es la que marca en gran medida el resto de las sesiones. Durante esta sesión se realizaron tres dinámicas grupales, relacionadas con la expresión corporal y el ocio. Gracias a ellas, el integrante es parte de un juego en el que adquiere confianza y se siente valorado. En dos de ellas se utiliza el contacto corporal como acercamiento entre los diferentes miembros de la acción, usando recursos lúdicos para el desarrollo de las mismas. En la última se introdujo por primera vez el componente artístico como medio para la presentación de cada uno de los participantes, utilizando pintura sobre un papel de gran formato que, una vez terminada, muestra un mural de recorridos vitales.

La participación de los internos y los educadores se desarrolló de manera abierta y horizontal, propiciando situaciones democráticas donde todos puedan mostrarse tal y cómo son y expresar libremente sus opiniones, reflexiones y puntos de vista.

El número de integrantes del taller fue variando. Las primeras sesiones fueron numerosas, y poco a poco se vio reducido debido a razones como desinterés en el planteamiento general o solapamiento con otras actividades de ocio.

El espacio en el que se desarrolla el taller consiste en una sala con mobiliario básico, que ya venía siendo utilizada como espacio para la creación artística, donde los internos se dedican sobre todo a construir piezas y objetos que mantienen expuestos en el aula. Entre estos trabajos predominaban espejos intervenidos con pintura y objetos artesanales como pulseras o carteras.

Tras varias sesiones en las que lo importante fue poner en común intereses, inquietudes y formas de entender lo que significa un taller artístico, se procedió a elaborar un plan flexible que organizaría los contenidos a tratar. Una vez valoradas y agrupadas estas inquietudes, se determina el siguiente bloque de contenidos:

- PINTURA EXPANDIDA: métodos de crear y entender composiciones pictóricasabiertas a la experimentación.

- INTERVENCIÓN DEL ESPACIO: abordar distintas problemáticas sociales desde las estrategias de intervención urbana.

- ARTE CORPORAL: abordar trabajos que integren el cuerpo como elemento principal en la acción artística.

- ORIGAMI: formas de trabajar el papel para crear formas figurativas.

Dependiendo del interés que se generase en cada uno de los bloques, así como de los ritmos de trabajo, se dedicarían más o menos sesiones a cada tema. 
REY \& SAINZ-RABANAL / Arte comunitario como herramienta de inclusión: experiencias en el Taller de Expresión Artística del Centro Penitenciario Madrid IV de Navalcarnero

Tabla 2

Cronograma de las actividades

CRONOGRAMA DE ACTIVIDADES 2015

\begin{tabular}{llccc}
\hline \multicolumn{5}{c}{ TOTAL DE SESIONES: 20 sesiones (febrero-junio; 5 meses) } \\
\hline \multicolumn{5}{c}{ DURACIÓN: 2 horas/sesión } \\
\hline MES 1 & MES 2 & MES 3 & MES 4 & MES 5 \\
\hline Planificación y trámites & 1 hora/semana aproximadamente \\
\hline Sesiones de presentación & 2 sesiones & & 5 sesiones & $\begin{array}{l}\text { Trabajo } \\
\text { autónomo de } \\
\text { los internos }\end{array}$ \\
\hline Pintura Expandida & 2 sesiones & 3 sesiones & 1 sesión \\
\hline $\begin{array}{l}\text { Carteles para la } \\
\text { intervención del espacio }\end{array}$ & 1 sesiones & 4 sesiones & 1 sesión \\
\hline Arte corporal & & & 1 sesión \\
\hline Origami & & & \\
\hline Sesión de evaluación grupal
\end{tabular}

Los métodos han sido en todas las sesiones similares. En primer lugar, se compartían en grupo algunas referencias visuales a modo de introducción del tema, construyendo los significados sobre las diferentes obras, las contradicciones, los posibles dobles sentidos, las características estéticas y la forma en que otro puede reapropiarse de esos conocimientos para la producción propia (fase teórica). Tras discutir en torno a los referentes presentados en cada sesión, se procedía a realizar una serie de actividades (fase práctica), mediante dinámicas y grupos abiertos de producción y colaboración. Al final de cada taller se ponía en común el trabajo realizado (fase de valoración) y, junto con las anotaciones del cuaderno de bitácora, en reuniones posteriores de los educadores se discutían las observaciones extraídas.

\section{- Sesiones de pintura expandida}

Las sesiones dedicadas a este tema fueron un total de cinco. Se comienza con la pintura puesto que resulta el tema más llamativo para los internos, abordándolo desde el concepto de "pintura expandida" y entendiéndolo como aquella disciplina que excede los límites previstos y se adentra en el terreno de otras disciplinas generando nuevos significados Rosalind Krauss (1985).

Los ejemplos artísticos que se presentan son composiciones pictóricas que se alejan de la pintura tradicional sobre lienzo. Estas jornadas se centraron sobre todo en ejercicios en los que priman los trampantojos, de modo que es el juego de perspectivas el que permite construir la imagen en su totalidad. Los principales trabajos sobre los que se construyó el debate fueron obras en espacios exteriores como las de George Rousse o el colectivo Boa Mistura.

Las primeras actividades prácticas consistieron en descubrir la pintura como 
herramienta de expresión, sobre todo con ejercicios en los que transformar motivos figurativos en abstractos. Un ejemplo fue llevar el autorretrato al plano abstracto, para luego clasificarlos grupalmente y volver a la materialización situando citas literarias según criterios individuales. Como actividad para producir composiciones pictóricas como reapropiación del espacio, se pusieron en marcha una serie de grupos de trabajo que desarrollaron diferentes propuestas para intervenir las paredes del propio espacio del taller. Tras el proceso de negociación a nivel burocrático, la pintura mural fue realizada con gran acogida por parte de los participantes, ya que la desarrollaron hasta su conclusión incluso fuera delos horarios del taller.

\section{- Sesiones sobre cartelería para la intervención en el espacio público}

Este tema se desarrolló durante diez sesiones, siendo el más extenso del taller. Se expusieron ejemplos visuales sobre cómo se construyen los carteles de cine, esto es, cómo el color, la composición y las representaciones componen una imagen para ofrecer una determinada percepción previa de la película. La actividad principal fue la creación de carteles de cine de manera individual, en el que cada uno debía convertirse en el protagonista de su propia historia. Muchos de los trabajos que salieron de esta actividad transmitían profundos sentimientos de soledad, lucha y resistencia. Con estos carteles se realizaron una serie de fotomontajes, creando simulacros para comprobar cómo resaltarían los trabajos de los internos en soportes como marquesinas de autobús, carteleras de cines y estructuras publicitarias.

En las siguientes jornadas se mostraron ejemplos de composiciones que unen texto e imagen, así como trabajos que utilizan el cartel para comunicar una denuncia o crítica concreta, como los materiales de las Guerrilla Girls. Se tomaron como referencia estas composiciones para producir una segunda ronda de carteles, los cuales sí saldrían al exterior para compartirse públicamente. Algunos de éstos hablaban sobre cómo sentimos la libertad, o la importancia de las pequeñas cosas y su significado personal.

En primer lugar, los carteles fueron colocados en dos puntos de la ciudad: la plaza del museo Reina Sofía de Madrid y el recinto del Museo del Prado. El grupo de educadores, junto a los carteles, atendía a las personas interesadas en ellos, explicando el origen de esos trabajos. Después los carteles se trasladaron por varios días a las paredes de la Facultad de Bellas Artes de la UCM, en concreto los que invitaban a intervenir y escribir sobre ellos. Por último, varios de los carteles se compartieron en redes sociales (Facebook), para conseguir mayor difusión y número de perspectivas por parte de personas interesadas. Tras exponer los trabajos se recogieron las opiniones y respuestas de las personas que los vieron para llevarlas al interior del centro y volver a discutirlas con los participantes.

\section{- Sesiones de Body Art y Origami}

Los dos bloques anteriores fueron los más extensos, ya que los trabajos en grupo se dilataron en el tiempo y las necesidades de las actividades se extendieron debido 
al interés que generaban. Por ello, los dos últimos temas consistieron en una sola sesión cada uno.

Con respecto al tema del arte corporal, se vieron algunas referencias, desde el bodypainting más comercial al más conceptual, debatiendo sobre técnicas más populares, como pueden ser el tatuaje o la henna. Se trabajó sobre todo con dinámicas grupales, de las que no se pudo obtener evidencia gráfica. Previamente a la sesión de evaluación se realizaron ejercicios de origami, viendo ejemplos de cómo llevar el trabajo en papel a una instalación artística y practicando algunas formas básicas como actividad relajante y de esparcimiento. En este caso tampoco se pudo evidenciar el trabajo con fotografías en el momento de la producción, y después los trabajos salieron de la prisión como regalo para familiares y amigos de los internos.

\section{Resultados obtenidos}

La extracción de datos para el análisis se llevó a cabo por tres medios: ficha de seguimiento, cuaderno de bitácora y documentación gráfica (fotografías, siempre que fue posible utilizar cámara dentro de las instalaciones).Estos instrumentos permiten analizar en qué medida se cumplieron los objetivos del taller. El análisis de la fichase presenta en la siguiente tabla:

Tabla 3.

Ficha de seguimiento actividades-objetivos

\begin{tabular}{|c|c|c|c|c|}
\hline \multirow[t]{2}{*}{ TEMÁTICA } & \multirow[t]{2}{*}{ ACTIVIDADES } & \multirow{2}{*}{$\begin{array}{l}\text { RESULTADOS } \\
\text { DEL TALLER }\end{array}$} & \multicolumn{2}{|c|}{ CUMPLIMIENTO DE OBJETIVOS } \\
\hline & & & $\begin{array}{l}\text { (1) Realizar } \\
\text { actividades que } \\
\text { conecten el mundo } \\
\text { de fuera y de dentro } \\
\text { del centro, a partir } \\
\text { de las estrategias } \\
\text { artísticas. }\end{array}$ & $\begin{array}{l}\text { (2) Ampliar el } \\
\text { imaginario hacia } \\
\text { propuestas y } \\
\text { producciones } \\
\text { de arte } \\
\text { contemporáneo } \\
\text { y análisis de la } \\
\text { cultura visual }\end{array}$ \\
\hline $\begin{array}{l}\text { INTERVENCIÓN } \\
\text { DEL ESPACIO } \\
P U ́ B L I C O\end{array}$ & $\begin{array}{l}\text { Visionado y debate } \\
\text { de contenidos. } \\
\text { Carteles. } \\
\text { Intervención mural } \\
\text { en el taller. }\end{array}$ & $\begin{array}{l}\text { Fotomontajes. } \\
\text { Dos } \\
\text { intervenciones } \\
\text { murales en la } \\
\text { sala-taller. }\end{array}$ & $\begin{array}{l}\text { Se logra a un nivel } \\
\text { alto, ya que los } \\
\text { trabajos salen al } \\
\text { exterior mediante } \\
\text { las redes sociales y } \\
\text { colocándolos en las } \\
\text { calles y vuelven con } \\
\text { feedback de gente } \\
\text { del exterior. }\end{array}$ & $\begin{array}{l}\text { Se consigue ya } \\
\text { que se logra } \\
\text { implementar lo } \\
\text { que se ve en los } \\
\text { contenidos en } \\
\text { el propio taller, } \\
\text { involucrando } \\
\text { al grupo } \\
\text { voluntariamente de } \\
\text { manera autónoma. } \\
\text { El objetivo se } \\
\text { logra a un nivel } \\
\text { alto. }\end{array}$ \\
\hline
\end{tabular}


REY \& SAINZ-RABANAL / Arte comunitario como herramienta de inclusión: experiencias en el Taller de Expresión Artística del Centro Penitenciario Madrid IV de Navalcarnero

\begin{tabular}{|c|c|c|c|c|}
\hline $\begin{array}{l}\text { PINTURA } \\
\text { EXPANDIDA }\end{array}$ & $\begin{array}{l}\text { - Visionado } \\
\text { y debate de } \\
\text { contenidos } \\
\text { - Ilustración- } \\
\text { presentación } \\
\text { - Autorretrato } \\
\text { abstracto. } \\
\text { - Intervención } \\
\text { mural en el taller. }\end{array}$ & $\begin{array}{l}\text { Fichas de } \\
\text { presentación } \\
\text { Mural colectivo } \\
\text { de autorretratos. }\end{array}$ & $\begin{array}{l}\text { Se consigue el } \\
\text { objetivo a un } \\
\text { nivel bajo-medio, } \\
\text { ya que no tiene } \\
\text { gran repercusión } \\
\text { en el exterior. Sin } \\
\text { embargo, sí que } \\
\text { sirve como vínculo } \\
\text { entre participantes } \\
\text { del taller (trabajo } \\
\text { colectivo) con los } \\
\text { otros reclusos no- } \\
\text { participantes que } \\
\text { se acercan a ver } \\
\text { los resultados, por } \\
\text { lo que supone un } \\
\text { traslado fuera del } \\
\text { propio espacio taller. }\end{array}$ & $\begin{array}{l}\text { Se presentaron } \\
\text { artistas como Boa } \\
\text { Mistura, George } \\
\text { Rousse, Igor } \\
\text { Eskinja, Guillermo } \\
\text { Mora, entre otros. } \\
\text { Las respuestas de } \\
\text { los participantes } \\
\text { son positivas, de } \\
\text { hecho, inspirados } \\
\text { por Rousse, se } \\
\text { realiza la obra- } \\
\text { intervención en el } \\
\text { taller. Por lo tanto, } \\
\text { existe una amplitud } \\
\text { de posibilidades de } \\
\text { creación artística } \\
\text { (de las expectativas } \\
\text { sobre realización de } \\
\text { pintura de paisaje } \\
\text { clásico a propuestas } \\
\text { más abstractas } \\
\text { y reflexivas).EI } \\
\text { objetivo se alcanza } \\
\text { a un nivel alto. }\end{array}$ \\
\hline BODYART & $\begin{array}{l}\text { Visionado y debate } \\
\text { de contenidos. }\end{array}$ & $\begin{array}{l}\text { No hay } \\
\text { resultados } \\
\text { prácticos. }\end{array}$ & $\begin{array}{l}\text { Objetivo no } \\
\text { conseguido. }\end{array}$ & $\begin{array}{l}\text { El objetivo } \\
\text { se logra a un } \\
\text { nivel medio. El } \\
\text { intercambio de } \\
\text { ejemplos, y la } \\
\text { relación que se } \\
\text { establece con otras } \\
\text { expresiones más } \\
\text { conocidas como el } \\
\text { tatuaje, hace que } \\
\text { el imaginario del } \\
\text { grupo se amplíe } \\
\text { hacia propuestas } \\
\text { más cotidianas. }\end{array}$ \\
\hline ORIGAMI & $\begin{array}{l}\text { Trabajos creativos } \\
\text { con papel con guías } \\
\text { de trabajo. }\end{array}$ & $\begin{array}{l}\text { Figuras de } \\
\text { origami } \\
\text { individuales. }\end{array}$ & $\begin{array}{l}\text { Se logra a un nivel } \\
\text { medio, ya que los } \\
\text { participantes en su } \\
\text { mayoría señalan } \\
\text { que los productos } \\
\text { servirán como } \\
\text { regalos para sus } \\
\text { visitantes familiares. } \\
\text { No hay documento } \\
\text { gráfico ya que los } \\
\text { trabajos salieron del } \\
\text { centro. }\end{array}$ & $\begin{array}{l}\text { Se presentan } \\
\text { algunos artistas } \\
\text { que trabajan con el } \\
\text { papel en diferentes } \\
\text { espacios como } \\
\text { Mademoiselle } \\
\text { Maurice o Nuria } \\
\text { Mora. En esta } \\
\text { sesión se denota } \\
\text { bastante interés por } \\
\text { aprender a realizar } \\
\text { estas figuras. } \\
\text { Además, se intuye } \\
\text { la cercanía con la } \\
\text { propuesta al ser } \\
\text { una actividad más } \\
\text { dirigida al concepto } \\
\text { de manualidad. Por } \\
\text { lo tanto, el objetivo } \\
\text { se alcanza a un } \\
\text { nivel medio-bajo. }\end{array}$ \\
\hline
\end{tabular}


En cuanto al primer objetivo, realizar actividades que conecten el mundo de fuera y de dentro del centro, a partir de las estrategias artísticas, las sesiones de intervención en el espacio público son las que consiguen este objetivo a un nivel más alto, ya que los trabajos realizados por los internos salen al exterior mediante las redes sociales de los educadores y/o colocándolos en las calles. Estos trabajos vuelven al taller con feedback del exterior, lo que promueve una reflexión entre la realidad dentro y fuera de prisión. Uno de los trabajos a destacar sería el cartel realizado con la pregunta “QQué es la libertad?”. La participación en las redes sociales fue alta, con un gran número de comentarios y acciones de compartir por parte de los usuarios, y sirvió para compararlas con las respuestas de los internos, que destacaron el valor de las acciones sencillas y cotidianas.

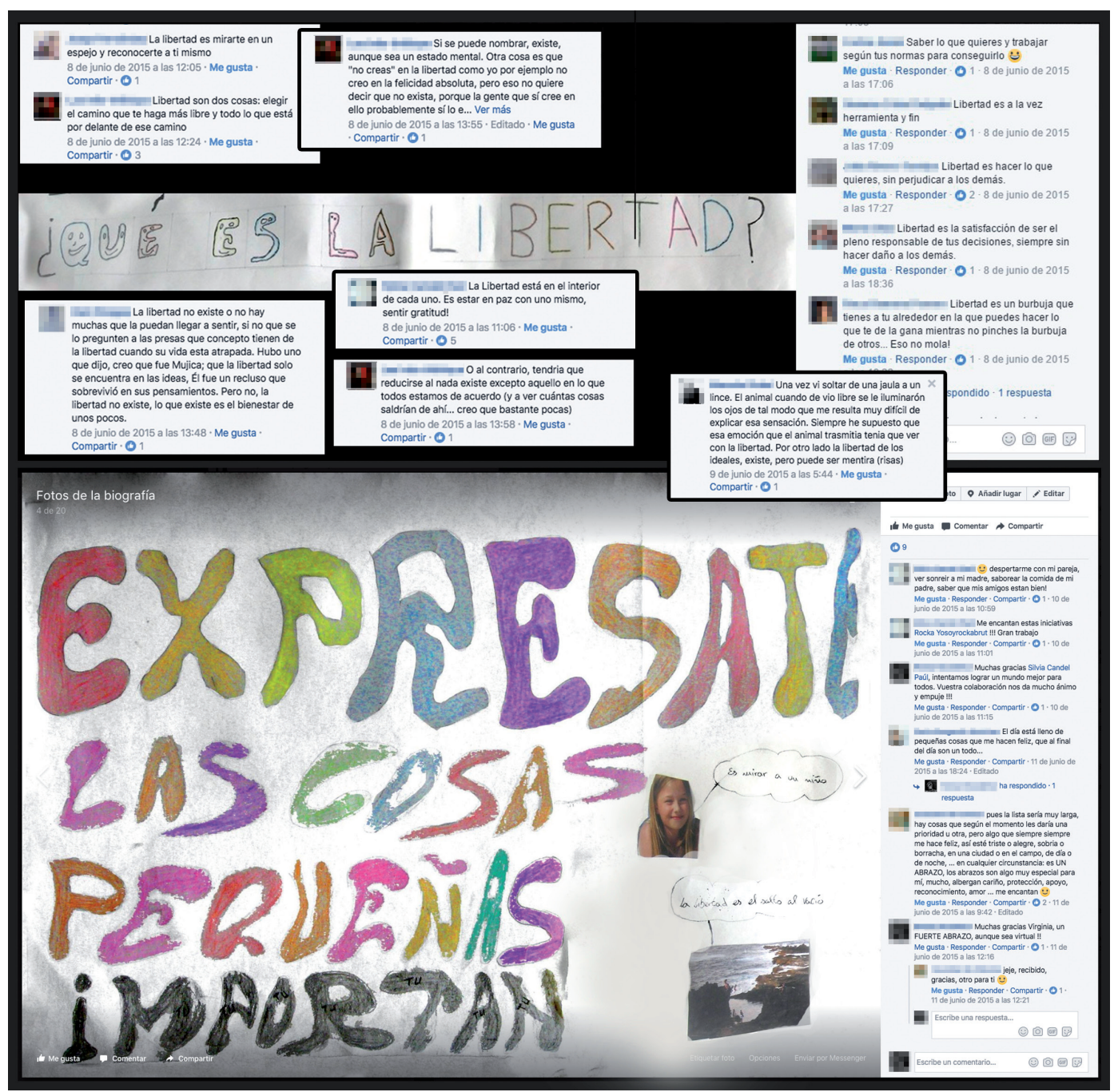

Figura 1.¿Qué es la libertad? Repercusión en las redes sociales. Fotografías de los autores 


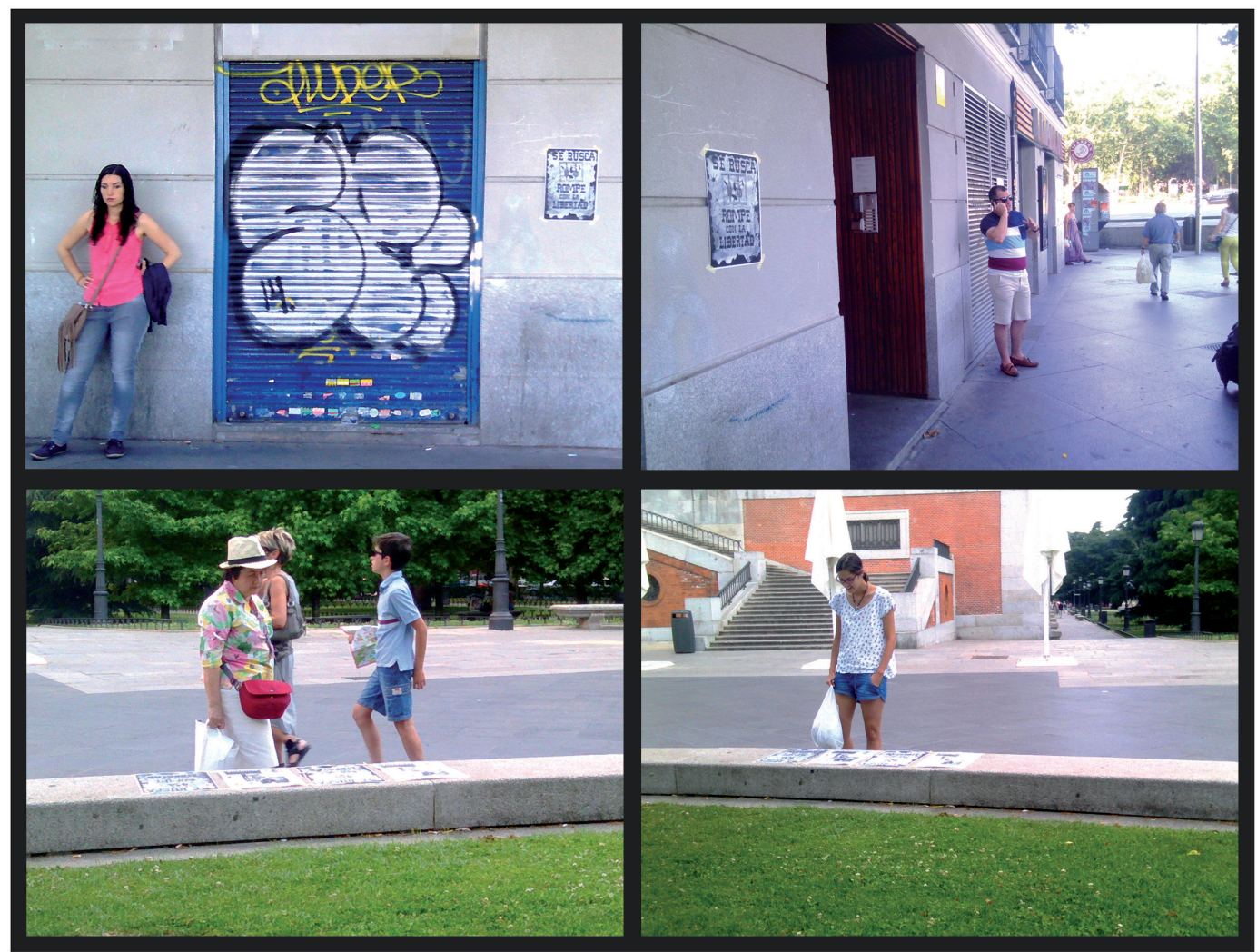

Figura 2. Trabajos del taller en el espacio público. Fotografías de los autores

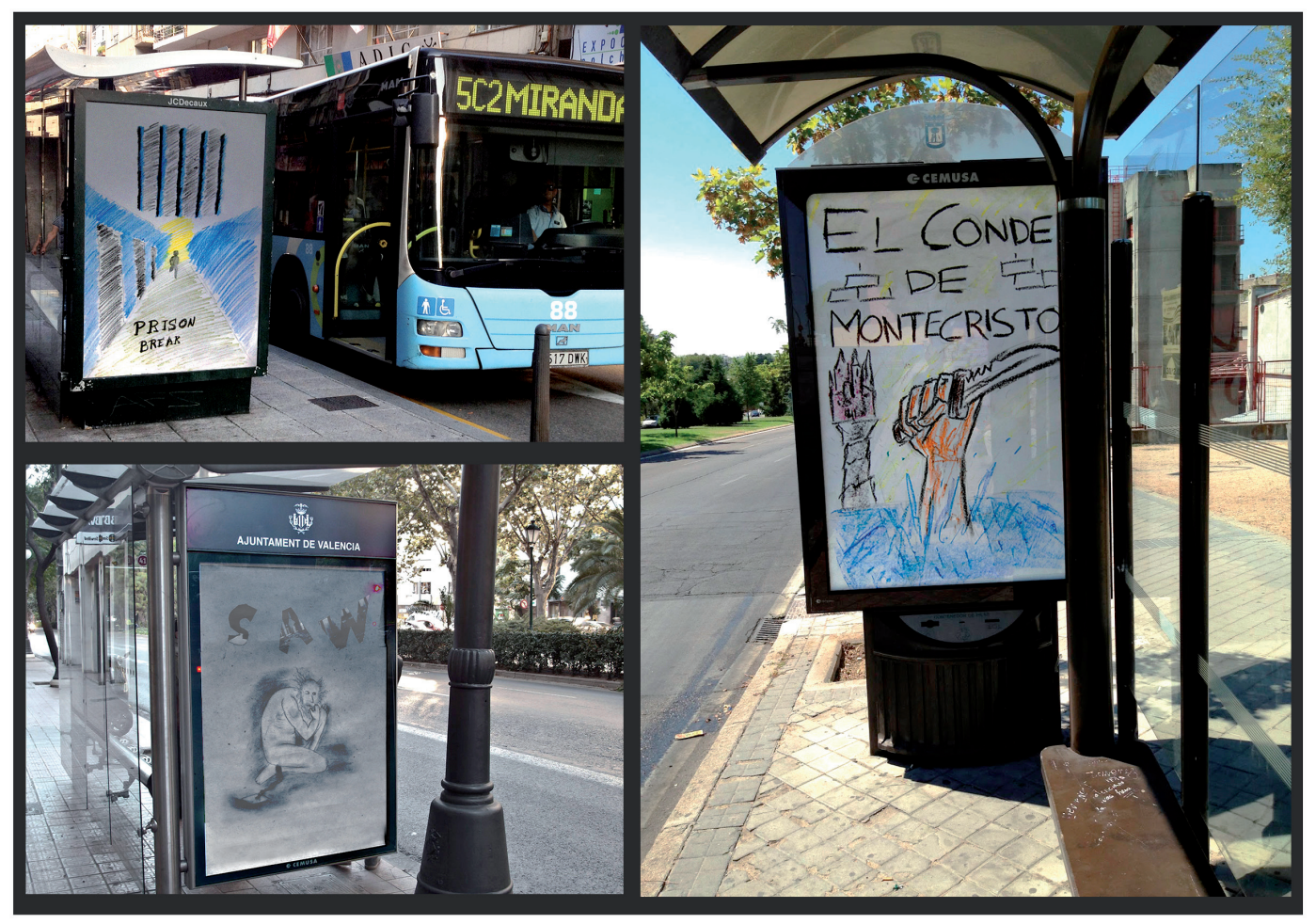

Figura 3.Trabajos del taller en el espacio público. Fotomontajes de los autores. 
El resto de sesiones tuvieron una conexión con el exterior bastante menor o nula. A destacar, las sesiones de pintura expandida, que culminaron con la realización de una obra en el aula del taller. Aunque no se consigue el objetivo propiamente dicho, sí que sirve como vínculo entre participantes del taller y los otros reclusos no participantes que se acercaron a ver los resultados con expectación, a la vez que los creadores les explicaban en qué consistía la intervención.

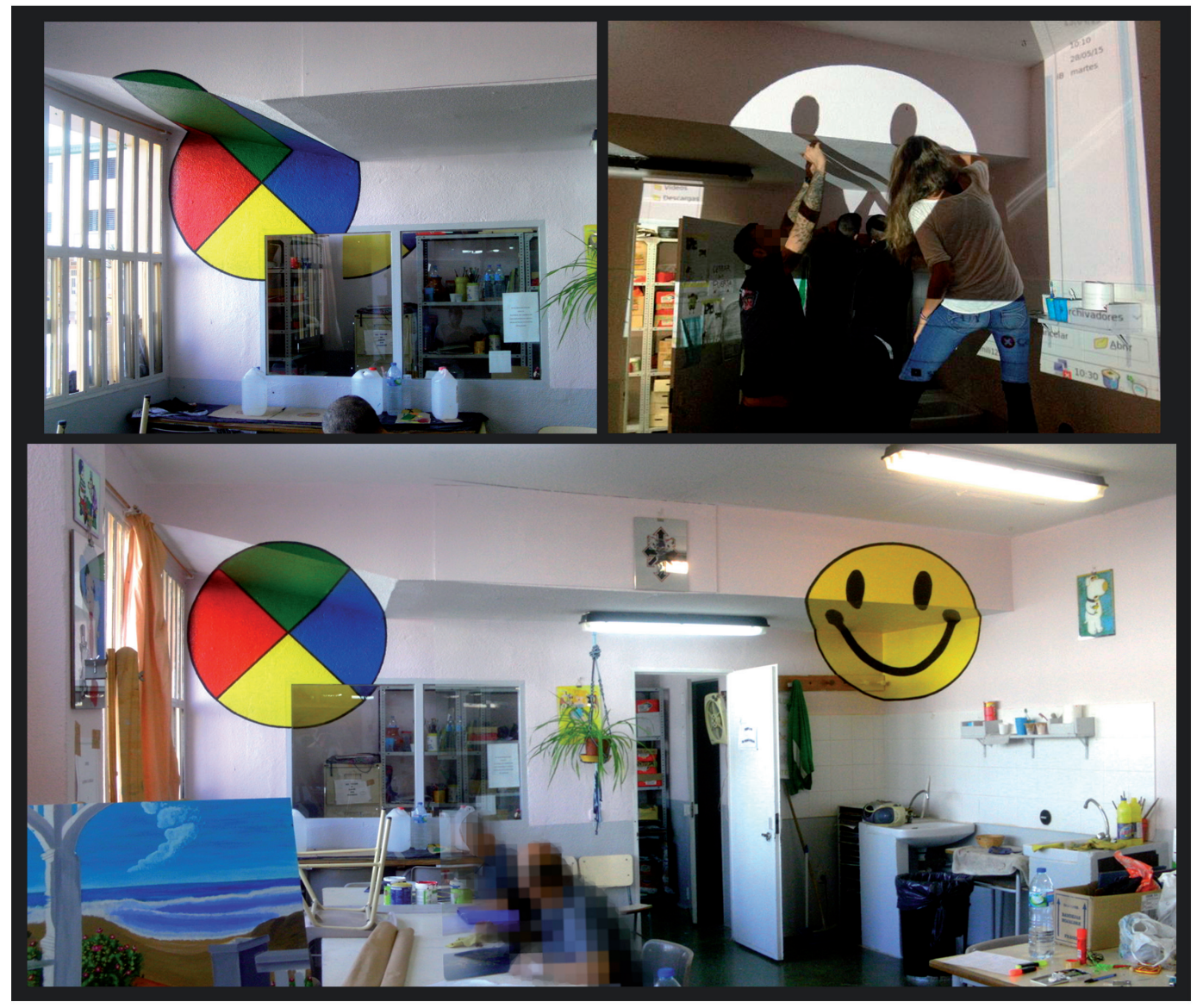

Figura 4. Intervenciones en la sala de taller. Fotografías de los autores

Relacionado con el segundo objetivo, que consistió en ampliar el imaginario hacia propuestas y producciones de arte contemporáneo y análisis de la cultura visual, las actividades que lo lograron a un nivel más alto fueron las que se desarrollaron bajo la temática de pintura expandida. Esto es debido a la semejanza con las expectativas que los internos expresaron en las primeras sesiones, entre las que sobresalía la producción pictórica. El ejercicio y la muestra de ejemplos que llevaban esa expectativa hacia propuestas más contemporáneas tuvieron una reacción muy positiva entre el grupo de participantes. 


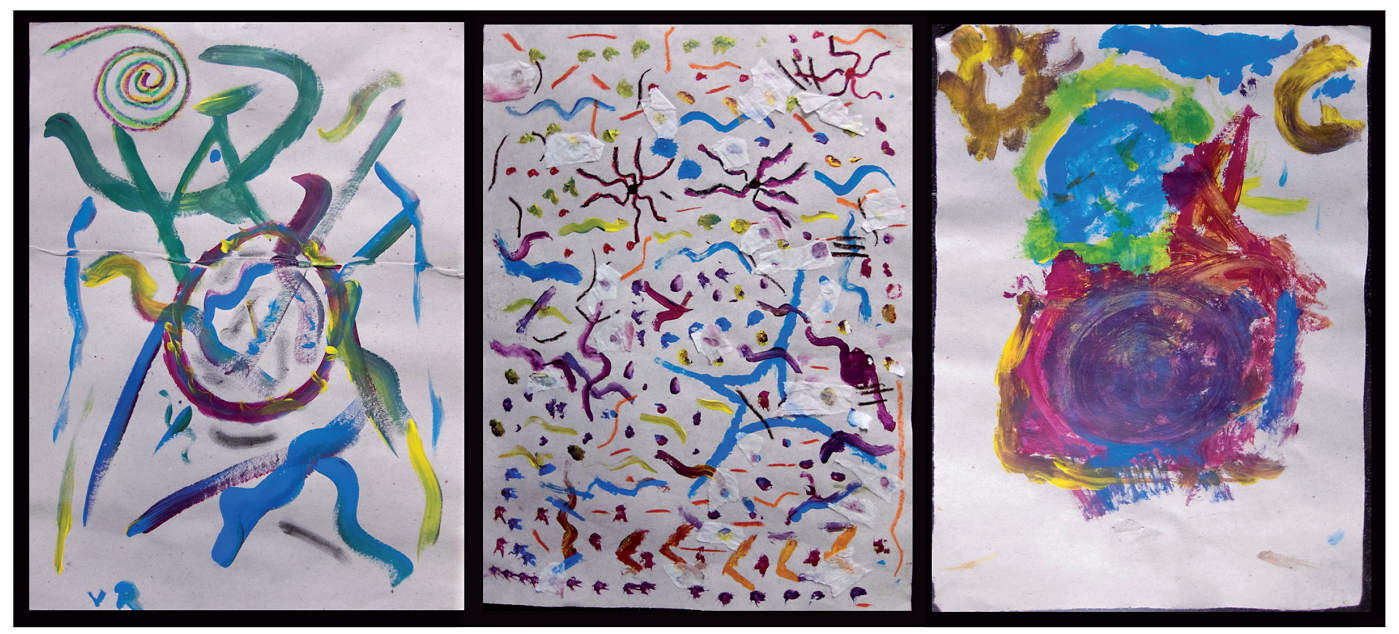

Figura 5.Autorretratos abstractos. Fotografías de los autores.

Otros resultados a destacar tienen que ver con las dinámicas humanas producidas en el taller, como el crecimiento personal de los asistentes o la creación de un espacio de libertad dentro de prisión. Los resultados en relación a estos elementos se recogen en el cuaderno de bitácora. En la sesión finalse planteó la pregunta ¿Qué has obtenido del taller? Las ideas más repetidas tenían que ver con la experiencia del taller; por un lado, una experiencia positiva - divertido (x3) y entretenido - y por otro, una experiencia novedosa - desconocido y sorpresa. Igualmente se hace hincapié en la duración del taller, con palabras como corto, veloz e intenso. Por último, aparecen palabras relacionadas con el desarrollo personal: crecimiento, reflexión, conocimiento, aprendizaje, compartir y empatizar.

En segundo lugar, se recogen en ese cuaderno algunas reflexiones sobre las expectativas previas al taller y cómo han cambiado durante su desarrollo. Algunas de ellas hacen referencia a las bajas expectativas iniciales: "Pocas expectativas al principio, me ha sorprendido mucho porque habéis venido con otras cosas", "Al principio hay incertidumbre hasta que tienes que hacer tu proyecto personal (...)", "Al principio no sabía lo que se iba a hacer y me pareció aburrido (...)". Otras reflexiones subrayan el objetivo del taller sobre la ampliación del imaginario artístico: "Muy bien los ejercicios de pintura expandida. No lo conocía y me va a venir muy bien", "He conocido otra forma de ver arte (...)", "[el arte expandido] tiene un sentido", "Me ha servido para aprender nuevas cosas". Y por último, se destacan los comentarios que hacen referencia al crecimiento personal y aprendizaje, en su mayoría positivos: "Me he sentido rejuvenecido (...)", "Mucha ilusión de aprender muchas cosas y he aprovechado el tiempo que he estado", "Ha sido una prueba $(. .)$.$" .$ 


\section{Conclusiones}

Las conclusiones que se extraen del proyecto y sus resultados tienen que ver con el nivel de cumplimiento de objetivos, así como con algunas observaciones finales.

Se puede señalar en primer lugar que el objetivo de la asociación coordinadora, Solidarios para el Desarrollo, fue una meta cumplida al crear en el taller un espacio de encuentro para el enriquecimiento de la realidad social de los implicados.

En relación al primer objetivo del taller, con el que se pretendía establecer mediante la producción artística una conexión entre el interior del centro penitenciario y el mundo exterior, se puede señalar que fueron las estrategias de producción de carteles que salían y entraban al centro las que lo consiguieron a un nivel más enriquecedor. El intercambio entre las opiniones de las personas ajenas al taller con los participantes internos supuso debates intensos en los que se evidenciaba la diferencia de sensaciones ante los mismos planteamientos. Por otro lado, la producción de figuras de origami funcionó bajo este mismo objetivo al producir objetos que salían del centro como regalo en las visitas de familiares. Estos productos, al ser más cercanos estética y procesualmente al concepto de "manualidad", se convierten fácilmente en objetos de intercambio entre seres queridos.

Las actividades de pintura expandida fueron las mejor aceptadas por los internos, siendo las que cumplieron a un mayor nivel el segundo objetivo, centrado en ampliar el imaginario en cuanto a las posibilidades de producción artística. Los ejercicios de pintura, al ser los más solicitados por el grupo, fueron un buen punto de partida para explorar otros modos de comprender esta disciplina. El uso específico del arte expandido, como disciplina híbrida que trabaja con aquellos aspectos del arte más alejados del imaginario previo, ha ayudado a ampliarlo y ha generado otros modos de relación con él. El desarrollo de una parte práctica en la que los participantes trasformasen su papel de espectadores a productores de imágenes artísticas ha sido un punto de inflexión a la hora de acercarse a estas otras prácticas, puesto que mediante su reafirmación como sujetos creativos han sido capaces de reflexionar sobre los propios contenidos y su posición como creadores.

Los resultados que se extraen del cuaderno, por otro lado, apuntan a conclusiones relacionadas con la experiencia que supuso el taller. Este proyecto se consideró por parte de los internos como una experiencia positiva y dinámica, a la vez que novedosa. De la misma manera, se transmitió por su parte la intensidad que supone un taller de este tipo a nivel afectivo, así como la sensación de que duró poco. La creación de un ambiente distendido, en el que los participantes pudiesen interactuar y experimentar ha ayudado claramente al desarrollo del programa, generando lazos y vínculos entre ellos y entre el grupo y los educadores. Si las relaciones entre educadores e internos son buenas, y se producen procesos de confianza, de libertad de expresión y de aprendizaje, sería beneficioso para estos contextos que 
REY \& SAINZ-RABANAL / Arte comunitario como herramienta de inclusión: experiencias en el Taller de Expresión Artística del Centro Penitenciario Madrid IV de Navalcarnero

los proyectos fueran a largo plazo, ocupando más de veinte sesiones de duración.

En relación con las sensaciones de desarrollo personal, como crecimiento, reflexión o aprendizaje, se coincide con lo expuesto por Villamarín (2004) al afirmar que los talleres creativos son positivos dentro de los centros penitenciarios, ya que a través de los mismos se consigue que los participantes desarrollen pensamiento crítico, generen autoconocimiento y se sientan valorados.

Se considera por último que este artículo repercute en el propio objeto de estudio, puesto que sirve como vehículo para dar a conocer este tipo de prácticas que se realizan en espacios de privación de libertad, y de esta forma conectan el interior de la prisión con el exterior.

\section{Referencias bibliográficas}

Aguilar, J., González, J., y Romero, J. (2009). Para el arte no hay rejas. La formación cultural como medio de reinserción social. Red visual, (9-10), 1-10. Recuperado de http://www.redvisual.net/pdf/9-10/carcel.pdf

Arias, L., Miracle, D., y Pérez, G. (2014). Tv Lab ADSIS. Los Kinze. Un proyecto de Neokinok.tv producido por LABoral Centro de Arte y Creación Industrial en colaboración con Fundación ADSIS. En M. Ruiz, y T. Vidal (Coord.), Arte, cárcel y cultura. Prácticas artísticas en Centros Penitenciarios (pp. 175-186). Barcelona: Cultura Sin Mesura.

Burgo, P. (2015, 11 de enero). El arte, un espacio de libertad en la cárcel. El Diario Norte. Recuperado de http://www.eldiario.es/norte/euskadi/arte-espacio-libertadcarcel_0_344015875.html

Cuesta, M. (2014, 8 de octubre). El arte de los presos. La Vanguardia. Recuperado de http://www.lavanguardia.com/cultura/20141008/54416866952/el-arte-de-lospresos.html

El sistema penitenciario español (2010). Secretaría General de Instituciones Penitenciarias. Madrid: Ministerio del Interior. Recuperado de. http://www. institucionpenitenciaria.es/web/export/sites/default/datos/descargables/ publicaciones/El_sistema_penitenciario_espanol.pdf

Felshin, N. (2001). ¿Pero esto es arte? El espíritu del arte como activismo. En P. Blanco, J. Carrillo, J. Claramonte, y M. Expósito, Modos de hacer: arte crítico, esfera pública y acción directa. (pp. 73-94). Salamanca: Universidad de Salamanca, Ediciones Universidad de Salamanca. 
García, J. (2014). Prólogo. En M. Ruiz, y T. Vidal (Coord.), Arte, cárcel y cultura. Prácticas artísticas en Centros Penitenciarios. Barcelona: Cultura Sin Mesura.

Gobierno de España (1978). Art. 25, Sección 1ª , Capítulo 2. Constitución Española.

Gobierno de España. Real Decreto 190/1996, de 9 de febrero, de Reglamento Penitenciario. BOE, 15 de febrero de 1996, núm. 40, p. 5380-5435.

Gómez, P. \& González, M. J. (2014). “Tiempo muerto”. Proyecto de colaboración con el grupo de internos de la cárcel de Palma. En M. Ruiz, y T Vidal (Coord.), Arte, cárcel y cultura. Prácticas artísticas en Centros Penitenciarios (pp. 98-106). Barcelona: Cultura Sin Mesura.

Health Development Agency. (2000). Art for Healt: A review of good practice in community-based arts projects and initiatives which impact on health and wellbeing .Londres: HDA

Hidalgo, M.A. (2011). Arte, intervención y acción social. La creatividad transformadora. En Arteterapia. Papeles de arteterapia y educación artística para la inclusión social, (6), 305-307.

Krauss, R. (1985). La escultura en el campo expandido. En H. Foster (Ed.), La posmodernidad (59-74). Barcelona: Kairós.

La Rueda Invertida (2014). (Pausa) Diálogos desde el Módulo 10. Madrid: Museo Nacional del Artes Decorativas.

Lago, M. E. (2014). Museos Públicos 25.2. En M. Ruiz, y T. Vidal (Coord.), Arte, cárcel y cultura. Prácticas artísticas en Centros Penitenciarios (pp. 125-132). Barcelona: Cultura Sin Mesura.

López, M. (2015). Indicadores sobre prácticas artísticas comunitarias: algunas reflexiones. Arteterapia-Papeles de arteterapia y educación artística para la inclusión social, (10), 209-234. Recuperado de http://revistas.ucm.es/index.php/ ARTE/article/view/51693

Machín, M. F. \& Talayero, S. (2014). Proyecto educativo de artium con el Centro Penitenciario de Nanclares. Proyecto colaborativo del proceso de educación de Artium y la EPA del País Vasco. En M. Ruiz, M., y T. Vidal (Coord.). Arte, cárcel y cultura. Prácticas artísticas en Centros Penitenciarios (pp. 153-162). Barcelona: Cultura Sin Mesura 
REY \& SAINZ-RABANAL / Arte comunitario como herramienta de inclusión: experiencias en el Taller de Expresión Artística del Centro Penitenciario Madrid IV de Navalcarnero

Moreno, A. (2012). La cárcel: ¿un tránsito hacia la reinserción a la vida activa? Algunas voces de los participantes en el curso de fotografía del Penal del Olmo (La Plata-Argentina). Arte, educación y cultura. Aportaciones desde la periferia. Jaén: COLBAA.

Observatorio del Tercer Sector de Bizkaia (2012). Arte para la inclusión y la transformación social. 3s_innovación. Recuperado de http://www.3sbizkaia.org/ Archivos/Documentos/Enlaces/1363_CAST-innovacion04.pdf

Palacios, A. (2009). El arte comunitario: origen y evolución de las prácticas artísticas colaborativas. Arteterapia - Papeles de arteterapia y educación artística para la inclusión social, (4), 197-211. Recuperado de http://revistas.ucm.es/index. $\mathrm{php/ARTE/article/view/9641}$

Revuelta, A. \& Arrizabalaga, A. (2014). Relat@s: proyecto ganador del Premio a un proyecto educativo de la Fundación PJ Miró, Mallorca (2008). En M. Ruiz, y T. Vidal (Coord.), Arte, cárcel y cultura. Prácticas artísticas en Centros Penitenciarios (pp. 88-97). Barcelona: Cultura Sin Mesura

Rodrigo, J. (2007). Educación artística y prácticas artístico-colaborativas: Territorios de cruce transversales. En Junta de Castilla y León (ed.), Arte contemporáneo y educación: un dialogo abierto. (pp. 76-96). Castilla y León: Consejería de Cultura y Turismo. Recuperado de http://javierrodrigomontero.blogspot.com/2010/04/ educacion-artistica-y-practicas.html

Ruiz, M. y Vidal, T. (Coord.) (2014). Arte, cárcel y cultura. Prácticas artísticas en Centros Penitenciarios. Barcelona: Cultura Sin Mesura.

Sola, B. (2014). Proyecto HIPATIA: Pedagogías de género en espacios de reclusión. En M. Ruiz, y T. Vidal (Coord.), Arte, cárcel y cultura. Prácticas artísticas en Centros Penitenciarios (pp. 163-174). Barcelona: Cultura Sin Mesura

Urtasun, A. (2014). Mi caja. Tu refugio. Oteiza desde el Centro Penitenciario de Pamplona. En Ruiz, M. \& Vidal, T. (Coord.). Arte, cárcel y cultura. Prácticas artísticas en Centros Penitenciarios (73-86). Barcelona: Cultura Sin Mesura.

Villamarín, E. (2004). Intervención artística en el medio penitenciario: el arte como reinserción social (Tesis doctoral). Universidad Complutense de Madrid. Recuperado de http://eprints.ucm.es/5462/

Web oficial del Colectivo Moraga: http:/www.colectivomoraga.com/sobrecolectivo-moraga 
REY \& SAINZ-RABANAL / Arte comunitario como herramienta de inclusión: experiencias en el Taller de Expresión Artística del Centro Penitenciario Madrid IV de Navalcarnero

Web oficial del DEAC MUSAC. Proyecto Hipatía: http://deacmusac.es/proyectohipatia-pedagogias-de-genero-en-espacios-de-reclusion

Web oficial de La Rueda Invertida: https://laruedainvertida.wordpress.com/

Web oficial de Solidarios para el Desarrollo: http://www.solidarios.org.es/ 